\title{
Sphingomonas mucosissima sp. nov. and Sphingomonas desiccabilis sp. nov., from biological soil crusts in the Colorado Plateau, USA
}

Correspondence Ferran Garcia-Pichel ferran@asu.edu

\section{G. S. N. Reddy and Ferran Garcia-Pichel}

School of Life Sciences, Arizona State University, Main Campus, Tempe, AZ 85287-4501, USA

Two bacterial strains, CP173-2 ${ }^{\top}$ and $C P 1 D^{\top}$, were isolated from biological soil crusts (BSCs) collected in the Colorado Plateau, USA. Both strains were pigmented, Gram-negative, non-motile rods and produced abundant mucus. They contained $\mathrm{C}_{16: 0}, \mathrm{C}_{18: 1} \omega 7 \mathrm{c}$ and $\mathrm{C}_{14: 0} 2-\mathrm{OH}$ as the predominant cellular fatty acids, ubiquinone-10 as the isoprenoid quinone and sphingoglycolipid. Based on the above characteristics, the isolates were assigned to the family Sphingomonadaceae; 16 rRNA gene signature nucleotides placed them within the genus Sphingomonas. Strains CP173-2 ${ }^{\top}$ and $C P 1 D^{\top}$ had a $16 \mathrm{~S}$ rRNA gene sequence similarity of $96.7 \%$ with each other and $91.6-98.9 \%$ sequence similarity with other species in the genus, indicating that they represent two separate, and possibly novel, species. The closest species to strains $\mathrm{CP} 173-2^{\top}$ and $\mathrm{CP}_{1} \mathrm{D}^{\top}$ were, respectively, Sphingomonas dokdonensis (98.9\% gene sequence similarity) and Sphingomonas panni (97.9\%). However, strain CP173-2 ${ }^{\top}$ exhibited a DNA-DNA relatedness of only $32.5 \%$ with the type strain of S. dokdonensis. Similarly, the DNA-DNA relatedness between strain $\mathrm{CP}_{1 \mathrm{D}^{\top}}$ and the type strain of S. panni was only $18 \%$. Phenotypic characterization supported this low relatedness. On the basis of this evidence, we propose that the new strains represent two novel species, for which the names Sphingomonas mucosissima sp. nov. (with type strain CP173- $2^{\top}=$ ATCC BAA $-1239^{\top}=$ DSM $17494^{\top}$ ) and Sphingomonas desiccabilis sp. nov. (with type strain $\mathrm{CP}_{1 D^{\top}}=\mathrm{ATCC} B A \mathrm{~B}-1041^{\top}=\mathrm{DSM} 16792^{\top}$ ) are proposed.
Biological soil crusts (BSCs) are formed on the topmost layers of the soil by the entanglement and gluing of mineral particles with micro-organisms and their extracellular polysaccharides (Belnap \& Lange, 2001). Most BSCs are dominated by cyanobacteria, with Microcoleus vaginatus and Microcoleus steenstrupii being the most common phototrophs in the Western arid lands of North America (Bowker et al., 2002; Garcia-Pichel, 2002; Gundlapally \& GarciaPichel, 2006; Nagy et al., 2005; Yeager et al., 2004). The heterotrophic microbial community of BSCs has only begun to be probed in recent years. Culture-dependent and independent studies on BSCs from the Colorado Plateau (Gundlapally \& Garcia-Pichel, 2006) and the Sonoran desert (Nagy et al., 2005) have clearly established the dominance of members of the genera Actinobacteria, Proteobacteria and

\section{Abbreviation: BSCs, biological soil crusts.}

The GenBank/EMBL/DDBJ accession number for the 16S rRNA gene sequences of strains CP173-2 ${ }^{\top}$ and $\mathrm{CP}^{\mathrm{D}} \mathrm{D}^{\top}$ are $\mathrm{AM} 229669$ and AJ871435, respectively.

A supplementary table detailing $16 \mathrm{~S}$ rRNA gene sequence similarities between strains CP173-2 and CP1D ${ }^{\top}$ and other species of the genus Sphingomonas is available in IJSEM Online.
Bacteriodetes among the bacterial chemoorganoheterotrophs and the widespread dominance of members of the genus Nitrosospira among bacterial ammonia-oxidizing chemoorganoautotrophs (Johnson et al., 2005). However, to date, only two descriptions of heterotrophic isolates from BSCs have been published, namely Dyadobacter crusticola (Reddy \& Garcia-Pichel, 2005) and Belnapia moabensis (Reddy et al., 2006). As a contribution to the study of biodiversity in these communities, we describe two additional isolates belonging to the genus Sphingomonas of the class Alphaproteobacteria.

The original genus Sphingomonas, as proposed by Yabuuchi et al. (1990) and emended by Takeuchi et al. (1993), was re-evaluated based on phylogenetic analysis, polyamine patterns, fatty acid profiling and nucleotide signature sequences (Pal et al., 2005, 2006; Takeuchi et al., 2001) and is now subdivided into four separate genera: Sphingomonas sensu stricto, Sphingobium, Novosphingobium and Sphingopyxis. Members of the genus Sphingomonas are yellow-pigmented, non-fermentative, Gram-negative, nonmotile or motile rods with a single polar flagellum and are characterized by the presence of a unique sphingoglycolipid with the long-chain base dihydrosphingosin, ubiquinone 10 
(Q-10), 2-hydroxymyristic acid (2-OH $\mathrm{C}_{14: 0}$ ) and the absence of 3-hydroxy fatty acids. Species of the genus Sphingomonas have great potential for biotechnological applications (Denner et al., 2001; Pollock, 1993).

Strains $\mathrm{CP}_{1} \mathrm{D}^{\mathrm{T}}$ and $\mathrm{CP} 173-2^{\mathrm{T}}$ were isolated from biological soil crust samples collected from sandy arid soils in the Colorado Plateau, USA ( $38^{\circ} 38^{\prime} 557^{\prime \prime} \mathrm{N} 109^{\circ} 38^{\prime} 910^{\prime \prime} \mathrm{W}$ and $38^{\circ} 09^{\prime} 905^{\prime \prime} \mathrm{N} 109^{\circ} 44^{\prime} 509^{\prime \prime} \mathrm{W}$ ), as described earlier (Reddy \& Garcia-Pichel, 2005). Orange $\left(\mathrm{CP} 173-2^{\mathrm{T}}\right)$ and yellow $\left(\mathrm{CP} 1 \mathrm{D}^{\mathrm{T}}\right)$ colonies of pigmented bacteria were picked up from BG11-PGY agar-solidified plates, purified by restreaking and maintained on $10 \times$ BG11-PGY plates. Morphological, biochemical characteristics and fatty acid methyl esters were analysed according to previously described methods (Reddy \& Garcia-Pichel, 2005; Reddy et al., 2006). Lipids were extracted and analysed as described by Suresh et al. (2004). Quinones were extracted according to the method of Collins et al. (1977) and separated by HPLC using the isocratic solvent system methanol/iso-propylether $(3: 1)$ (Tamaoka et al., 1983; Tamaoka, 1986). Sphingomonas asaccharolytica ATCC $51839^{\mathrm{T}}$, Sphingomonas panni DSM $15761^{\mathrm{T}}$ and Sphingomonas dokdonensis DS- $4^{\mathrm{T}}$ were used as standards for identifying the lipids and quinones. These strains were also used for DNA-DNA hybridization with the two novel strains using the filter hybridization method described by Pandey et al. (2002). 16S rRNA gene sequencing and phylogenetic analysis were performed as described previously (Kimura, 1980; Kumar et al., 2001; Reddy \& Garcia-Pichel, 2005; Thompson et al., 1994).

Cells of strains $\mathrm{CP} 173-2^{\mathrm{T}}$ and $\mathrm{CP} 1 \mathrm{D}^{\mathrm{T}}$ are Gram-negative, rod-shaped (Fig. 1), non-motile, mucoid and pigmented and contain $\mathrm{C}_{16: 0}, \mathrm{C}_{18: 1} \omega 7 c$ and $\mathrm{C}_{14: 0} 2-\mathrm{OH}$ as the major cellular fatty acids, as well as ubiquinone-10 as the isoprenoid quinone. A comparison of the lipid profiles of strains $\mathrm{CP} 1 \mathrm{D}^{\mathrm{T}}$ and $\mathrm{CP} 173-2^{\mathrm{T}}$ with those of S. asaccharolytica ATCC $51839^{\mathrm{T}}$ indicated that they contain, among other lipids, abundant sphingoglycolipid. These traits would place both novel isolates within the family Sphingomonadaceae. BLAST similarity analysis based on 16S rRNA gene sequences placed both novel strains clearly within the genus Sphingomonas. In addition, their gene sequences contained the signature sequences $\mathrm{C}-\mathrm{G}, \mathrm{G}, \mathrm{G}, \mathrm{G}-\mathrm{C}, \mathrm{U}-\mathrm{G}, \mathrm{U}$, $\mathrm{U}, \mathrm{U}, \mathrm{U}, \mathrm{A}, \mathrm{C}, \mathrm{G}$ and $\mathrm{C}$ at positions 52-359 (based on the Escherichia coli numbering system), 134, 593, 987-1218, 990-1215, 412, 562, 748, 823, 877, 841, 1438 and 1463, respectively, which are characteristic of the genus (Maruyama et al., 2006; Takeuchi et al., 2001). The two novel strains shared a $16 \mathrm{~S}$ rRNA gene sequence similarity of $96.7 \%$ (see Supplementary Table S1 in IJSEM Online), confirming that they represent separate species belonging to the genus Sphingomonas (Stackebrandt \& Goebel, 1994). In addition, strain $\mathrm{CP}_{1} \mathrm{D}^{\mathrm{T}}$ is conspicuously different from strain $\mathrm{CP} 173-2^{\mathrm{T}}$ in that it is yellow-pigmented, whereas strain CP173-2 $2^{\mathrm{T}}$ is orange-pigmented; other differentiating characteristics are listed in Table 1.

Strain $\mathrm{CP}_{1 \mathrm{D}^{\mathrm{T}}}$ shared a sequence similarity of $92-97.9 \%$ with other members of the genus Sphingomonas (Supplementary Table S1); its closest relative was S. panni (97.9\% gene sequence similarity). In addition, the topologies of the $16 \mathrm{~S}$ rRNA gene sequence phylogenetic trees, constructed using the unweighted pair group method with arithmetic mean (UPGMA), neighbour-joining (NJ), maximumevolution (ME) and DNA parsimony methods, all strongly supported the affiliation of strain $\mathrm{CP}_{1} \mathrm{D}^{\mathrm{T}}$ with $S$. panni (Busse et al., 2005) (Fig. 2). However, DNA-DNA hybridization between strain $\mathrm{CP}_{1 \mathrm{D}}^{\mathrm{T}}$ and S. panni was only $18 \%$ indicating a clear species separation. This was further confirmed by the phenotypic differences that strain $\mathrm{CP}_{1} \mathrm{D}^{\mathrm{T}}$ exhibited when compared with S. panni (Tables 1 and 2). The differences between these strains were particularly marked with respect to the fatty acid profiles as strain $\mathrm{CP} 1 \mathrm{D}^{\mathrm{T}}$ contained higher amounts of $\mathrm{C}_{17: 1} \omega 6 c$ and $\mathrm{C}_{18: 1} \omega 5 c$ and significantly lower amounts of the major fatty acid $\mathrm{C}_{18: 1} \omega 7 c$. Therefore, based on the above characteristics, strain $\mathrm{CP} 1 \mathrm{D}^{\mathrm{T}}$ was assigned as the type strain of a novel species, Sphingomonas desiccabilis sp. nov.

Strain CP173-2 ${ }^{\mathrm{T}}$ showed a $16 \mathrm{~S}$ rRNA gene sequence similarity of $91.6-98.9 \%$ with other species of the genus Sphingomonas (see Supplementary Table S1). The most similar species, with $98.9 \%$ gene sequence similarity, was $S$.
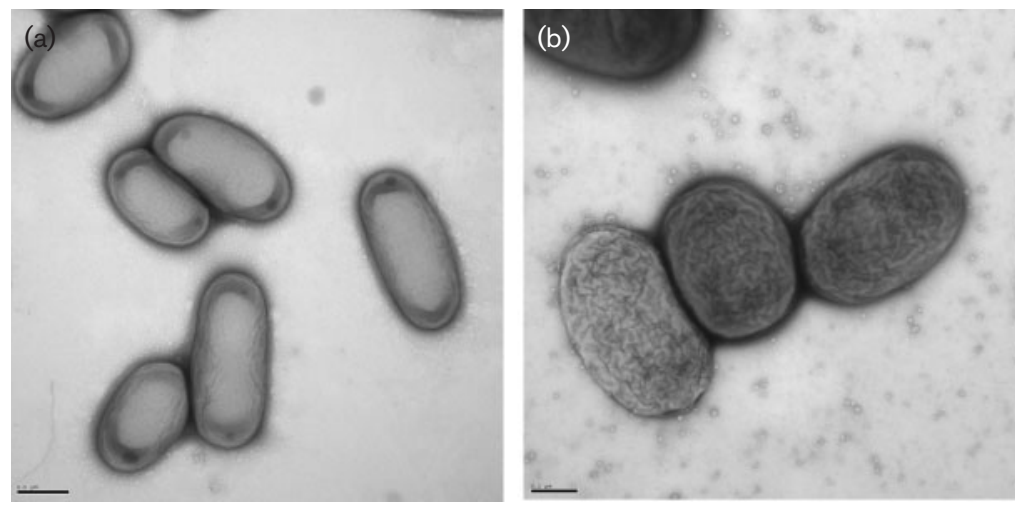

Fig. 1. Transmission electron micrograph of negatively stained cells of (a) Sphingomonas mucosissima CP173-2 ${ }^{\top}$ and (b) Sphingomonas desiccabilis $\mathrm{CP}_{1} \mathrm{D}^{\top}$. Note the extracellular mucus as tenuous shadowing around cells. Bars, $1 \mu \mathrm{m}$. 
Table 1. Phenotypic characteristics that differentiate strains $C P 1 D^{\top}$ and $C P 173-2^{\top}$ from related species of the genus Sphingomonas

Taxa: 1, Sphingomonas desiccabilis sp. nov. CP1D ${ }^{\mathrm{T}}$; 2, S. panni DSM $15761^{\mathrm{T}}$ (data from Busse et al., 2005); 3, Sphingomonas mucosissima sp. nov. CP173-2 $2^{\mathrm{T}}$; 4, S. dokdonensis DS-4 ${ }^{\mathrm{T}}$ (Yoon et al., 2006); 5, S. asaccharolytica Y-345 ${ }^{\mathrm{T}}$ (Takeuchi et al., 1995); 6, Sphingomonas pruni Y-250 ${ }^{\mathrm{T}}$ (Takeuchi et al., 1995); 7, Sphingomonas mali Y-351 ${ }^{\mathrm{T}}$ (Takeuchi et al., 1995); 8, Sphingomonas melonis DSM 14444 ${ }^{\mathrm{T}}$ (Buonaurio et al., 2002); 9, Sphingomonas aquatilis JSS7 $^{\mathrm{T}}$ (Lee et al., 2001); 10, Sphingomonas koreensis JSS26 ${ }^{\mathrm{T}}$ (Lee et al., 2001); 11, Sphingomonas abaci DSM $15867^{\mathrm{T}}$ (Busse et al., 2005); 12, Sphingomonas faeni DSM 14747 ${ }^{\mathrm{T}}$ (Busse et al., 2003); 13, Sphingomonas aerolata DSM 14746 ${ }^{\mathrm{T}}$ (Busse et al., 2003); 14, Sphingomonas aurantiaca DSM $14748^{\mathrm{T}}$ (Busse et al., 2003); 15, Sphingomonas oligophenolica CIP $107926^{\mathrm{T}}$ (Ohta et al., 2004); 16, Sphingomonas echinoides DSM $1805^{\mathrm{T}}$ (Denner et al., 1999). Y, Yellow; LY, light yellow; O, orange; V, variable; NG, not grown; ND, not determined; NR, not reported; + , positive; - , negative; $(+)$, weakly positive.

\begin{tabular}{|c|c|c|c|c|c|c|c|c|c|c|c|c|c|c|c|c|}
\hline Characteristic & 1 & 2 & 3 & 4 & 5 & 6 & 7 & 8 & 9 & 10 & 11 & 12 & 13 & 14 & 15 & 16 \\
\hline Temperature range for growth $\left({ }^{\circ} \mathrm{C}\right)$ & $15-37$ & $15-37$ & $15-28$ & $10-34$ & NR & NR & NR & NR & NR & NR & $15-28$ & $5-28$ & $5-28$ & $5-28$ & $5-37$ & NR \\
\hline Motility & - & - & - & + & - & - & + & - & + & + & - & + & + & + & - & + \\
\hline Colour & $\mathrm{Y}$ & $\mathrm{Y}$ & $\mathrm{O}$ & $\mathrm{Y}$ & $\mathrm{Y}$ & $\mathrm{Y}$ & LY & $\mathrm{Y}$ & $\mathrm{Y}$ & $\mathrm{Y}$ & $\mathrm{O}$ & $\mathrm{O}$ & $\mathrm{O}$ & $\mathrm{O}$ & $\mathrm{Y}$ & Y \\
\hline \multicolumn{17}{|l|}{ Biochemical characteristics: } \\
\hline Oxidase & + & - & + & + & + & + & + & + & + & + & - & + & + & + & + & + \\
\hline Lipase & + & NR & NG & + & + & + & + & - & NR & NR & NR & - & - & $\mathrm{V}$ & NR & - \\
\hline Gelatinase & + & NR & - & + & - & - & - & - & - & - & NR & - & - & - & + & - \\
\hline$\beta$-Galactosidase & + & + & - & - & + & + & + & + & + & + & + & + & + & + & NR & + \\
\hline Nitrate reduction & + & NR & - & - & - & - & - & - & - & - & NR & - & - & - & + & + \\
\hline \multicolumn{17}{|l|}{ Hydrolysis of: } \\
\hline Starch & + & NR & + & + & + & + & - & - & NR & NR & NR & - & - & - & - & + \\
\hline Aesculin & - & + & - & + & + & + & + & + & + & + & + & + & + & + & NR & + \\
\hline \multicolumn{17}{|l|}{ Assimilation of carbon compounds: } \\
\hline Acetate & - & NR & + & + & - & - & - & NR & NR & NR & NR & + & + & + & + & - \\
\hline Alanine & - & - & + & NR & + & + & - & - & NR & NR & - & + & + & + & NR & - \\
\hline Arabinose & + & - & + & - & + & + & + & + & + & - & - & + & + & + & + & + \\
\hline Aspartic acid & - & - & - & NR & + & - & + & - & NR & NR & - & + & - & - & NR & - \\
\hline Cellobiose & + & - & + & + & - & + & + & + & NR & NR & - & + & + & + & + & + \\
\hline Citrate & - & + & - & - & - & - & - & - & - & - & - & + & $\mathrm{V}$ & + & + & - \\
\hline Fructose & - & - & + & - & - & - & + & + & NR & NR & - & + & + & + & - & - \\
\hline Fumarate & - & + & - & NR & $(+)$ & + & + & + & NR & NR & + & + & + & + & NR & NR \\
\hline Galactose & + & - & + & - & - & + & + & + & NR & NR & - & + & + & + & + & + \\
\hline Glucose & + & - & + & - & + & + & + & + & + & + & - & + & + & + & + & + \\
\hline Glutamic acid & - & NR & - & - & + & + & + & + & NR & NR & NR & NR & NR & NR & NR & - \\
\hline Lactose & - & - & - & NR & $(+)$ & + & + & - & NR & NR & - & NR & $\mathrm{NR}$ & NR & + & ND \\
\hline Leucine & - & - & - & NR & - & - & - & NR & NR & NR & - & + & - & + & NR & - \\
\hline Maltose & + & + & - & - & + & + & + & + & + & + & - & + & + & + & + & + \\
\hline Mannitol & - & - & - & - & + & - & - & - & NR & NR & - & - & - & - & NR & - \\
\hline Mannose & + & + & - & + & + & + & + & + & - & - & - & + & + & + & + & - \\
\hline Melibiose & + & - & + & NR & + & $(+)$ & + & - & - & + & - & + & $\mathrm{V}$ & + & NR & - \\
\hline Proline & + & + & - & NR & + & - & - & + & NR & NR & - & - & - & + & NR & - \\
\hline Pyruvate & + & + & + & + & - & - & - & NR & NR & NR & - & + & $\mathrm{V}$ & + & + & - \\
\hline Raffinose & + & - & - & NR & - & - & + & - & NR & NR & - & NR & NR & NR & + & ND \\
\hline Rhamnose & + & - & - & NR & $(+)$ & + & + & + & + & + & - & + & - & - & NR & - \\
\hline Ribose & + & - & + & NR & $\mathrm{NR}$ & NR & NR & - & - & - & - & - & - & - & NR & - \\
\hline Sucrose & + & - & + & - & $(+)$ & + & + & + & + & + & - & + & + & + & NR & - \\
\hline Trehalose & - & + & - & + & $(+)$ & + & + & + & NR & NR & - & + & + & + & NR & + \\
\hline Xylose & + & - & + & - & - & + & + & + & NR & NR & - & + & + & + & + & - \\
\hline
\end{tabular}

dokdonensis (Yoon et al., 2006), suggesting that strain CP173-2 ${ }^{\mathrm{T}}$ could be a further strain of $S$. dokdonensis. Further, the topologies of the phylogenetic trees constructed using different methods suggested a strong affiliation (as supported by the bootstrap values of $88-99 \%$ ) of strain
CP173-2 ${ }^{\mathrm{T}}$ to $S$. dokdonensis (Fig. 2). However, low relatedness $(32.5 \%)$ in DNA-DNA hybridization experiments between strain CP173-2 ${ }^{\mathrm{T}}$ and the type strain of $S$. dokdonensis, indicates that the strains represent separate species. In addition, strain CP173-2 ${ }^{\mathrm{T}}$ and $S$. dokdonensis exhibited 


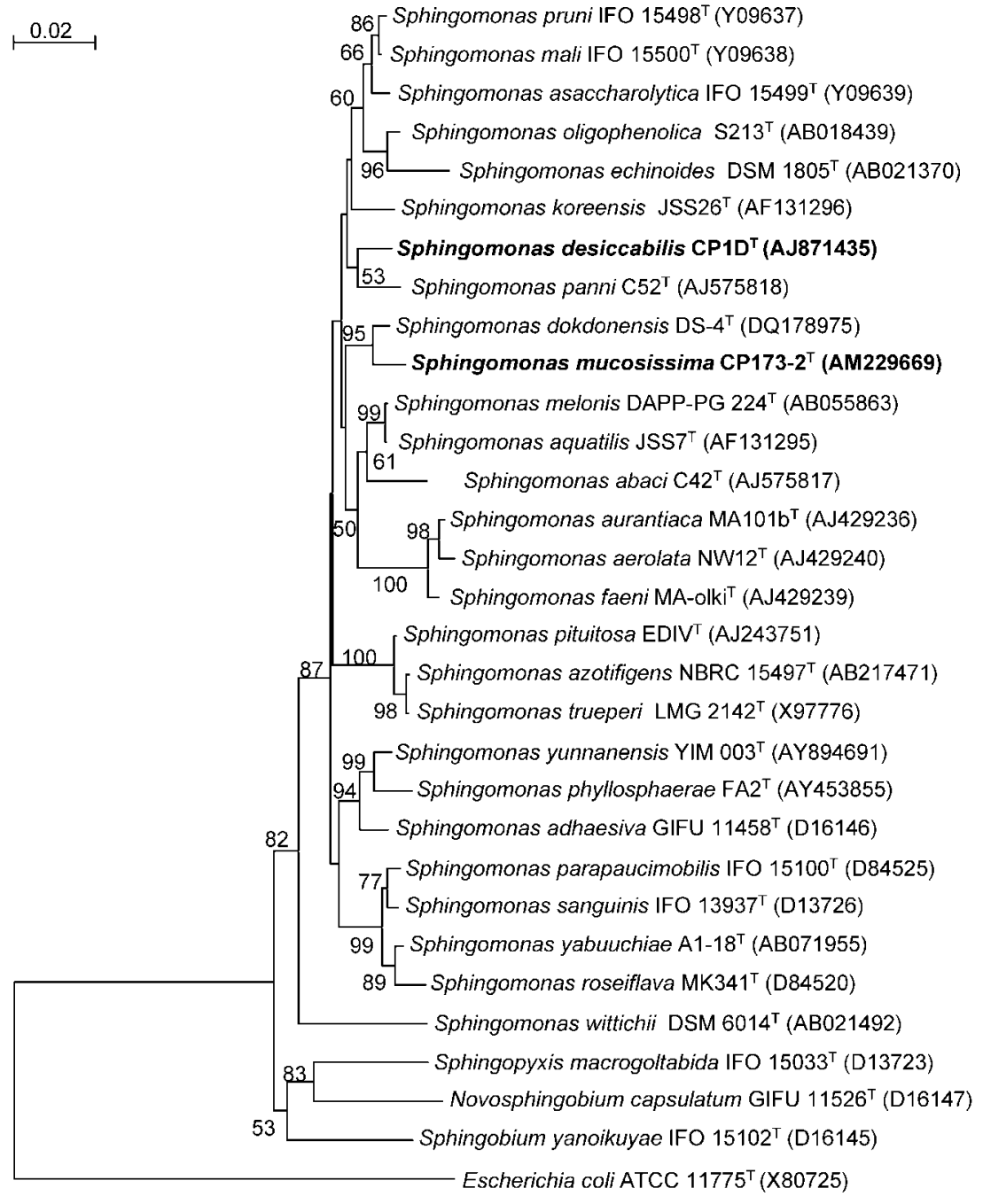

Fig. 2. Neighbour-joining tree based on 16S rRNA gene sequences showing the phylogenetic relationship between Sphingomonas mucosissima sp. nov. CP173-2 ${ }^{\top}$, Sphingomonas desiccabilis sp. nov. CP1D ${ }^{\top}$ and other related micro-organisms. Bootstrap values (expressed as a percentage of 1000 replications) $>50 \%$ are given at the nodes. Bar, 0.02 substitutions per nucleotide position. several phenotypic differences (morphological and biochemical as well as chemotaxonomic; Tables 1 and 2), further supporting the species separation. Most remarkably, strain $\mathrm{CP} 173-2^{\mathrm{T}}$ is unique when compared to its closest relatives as it gives a negative result in tests for $\beta$-galactosidase and has high proportion of $\mathrm{C}_{14: 0}$ and $\mathrm{C}_{16: 0} 2-\mathrm{OH}$. Thus, based on the cumulative differences that strain CP173-2 ${ }^{\mathrm{T}}$ exhibited when compared with the recognized species of the genus Sphingomonas, we suggest that strain CP173-2 $2^{\mathrm{T}}$ represents a novel species, for which the name Sphingomonas mucosissima sp. nov. is proposed.

It appears that the strains $\mathrm{CP} 1 \mathrm{D}^{\mathrm{T}}$ and $\mathrm{CP} 173-2^{\mathrm{T}}$ could impart some stability to the BSCs they inhabit as they produce very abundant extrapolysaccharides, as evidenced in their mucous appearance in transmission electron micrographs (Fig. 1). Given their extreme environment of origin, these strains are likely to be able to withstand repeated and severe desiccation as well as exposure to temperature extremes and solar radiation. This may make them valuable in biotechnology or bioremediation applications tailored to extreme conditions.

\section{Description of Sphingomonas mucosissima sp. nov.}

Sphingomonas mucosissima (mu.co.sis'si.ma. L. fem. superlative adj. mucosissima slimiest, referring to the appearance of the strains).

Colonies are orange-pigmented, extremely mucoid, convex, round and smooth. Cells are Gram-negative, non-motile rods of $0.75 \mu \mathrm{m}$ diameter and $1.7 \mu \mathrm{m}$ in length. Grows at $15-30{ }^{\circ} \mathrm{C}$, but not at $37^{\circ} \mathrm{C}$, with an optimum growth temperature of $25^{\circ} \mathrm{C}$ and a pH of 7. Can tolerate up to $2.5 \%$ $\mathrm{NaCl}$. Cells are positive for catalase, oxidase, phosphatase and do not grow on test media plates of lipase and urease. Negative result in tests for $\beta$-galactosidase, gelatinase, lysine decarboxylase, ornithine decarboxylase, phenylalanine deaminase and arginine dihydrolase activities. Can hydrolyse starch, but not aesculin. Does not reduce nitrate to nitrite and cannot produce $\mathrm{H}_{2} \mathrm{~S}$ gas. Negative results in methyl red, Voges-Proskauer, indole and Simmons' citrate tests. Can utilize acetate, L-arabinose, D-cellobiose, glucose, D-fructose, D-galactose, D-glucose, glycerol, inulin, D-melibiose, 
Table 2. Comparison of fatty acid contents (\%) of strains $C P 173-2^{\top}$ and $C P 1 D^{\top}$ with other related species of the genus Sphingomonas

Taxa: 1, Sphingomonas mucosissima CP173-2 $2^{\mathrm{T}}$; 2, S. dokdonensis DS-4 ${ }^{\mathrm{T}}$ (Yoon et al., 2006); 3, Sphingomonas desiccabilis CP1D ${ }^{\mathrm{T}}$; 4, S. panni DSM $15761^{\mathrm{T}}$ (Busse et al., 2005); 5, S. asaccharolytica Y-345 ${ }^{\mathrm{T}}$ (Denner et al., 1999); 6, S. pruni Y-250 ${ }^{\mathrm{T}}$ (Denner et al., 1999); 7, S. mali Y$351^{\mathrm{T}}$ (Denner et al., 1999); 8, S. melonis DAPP-PG 224 (Buonaurio et al., 2002); 9, Sphingomonas aquatilis JSS7 ${ }^{\mathrm{T}}$ (Lee et al., 2001); 10,

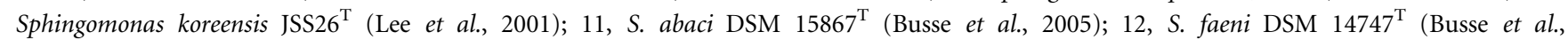
2003); 13, S. aerolata DSM 14746 ${ }^{\mathrm{T}}$ (Busse et al., 2003); 14, S. aurantiaca DSM $14748^{\mathrm{T}}$ (Busse et al., 2003); 15, S. oligophenolica CIP $107926^{\mathrm{T}}$ (Ohta et al., 2004); 16, S. echinoides DSM $1805^{\mathrm{T}}$ (Denner et al., 1999). +, Detected; ND, not detected; NR, not reported.

\begin{tabular}{|c|c|c|c|c|c|c|c|c|c|c|c|c|c|c|c|c|}
\hline Fatty acid & 1 & 2 & 3 & 4 & 5 & 6 & 7 & $8^{*}$ & 9 & 10 & 11 & 12 & 13 & 14 & 15 & 16 \\
\hline $\mathrm{C}_{14: 0}$ & 10.3 & 0.8 & 0.8 & 1.1 & ND & ND & ND & NR & 0.7 & 1.9 & ND & 2.0 & 1.9 & 3.0 & 3.0 & 0.9 \\
\hline $\mathrm{C}_{15: 0}$ & 1.4 & ND & 0.9 & 1.1 & ND & ND & ND & NR & $\mathrm{ND}$ & 0.2 & $\mathrm{ND}$ & $\mathrm{ND}$ & 0.5 & 0.8 & ND & $\mathrm{ND}$ \\
\hline $\mathrm{C}_{17: 0}$ & ND & ND & 0.7 & $\mathrm{ND}$ & ND & ND & ND & NR & ND & ND & $\mathrm{ND}$ & ND & ND & 0.2 & ND & $\mathrm{ND}$ \\
\hline $\mathrm{C}_{18: 0}$ & 4.1 & ND & 6.1 & $\mathrm{ND}$ & ND & ND & ND & NR & 1.0 & 0.6 & ND & ND & ND & 0.3 & ND & ND \\
\hline $\mathrm{C}_{17: 1} \omega 6 c$ & 2.7 & ND & 6.8 & 3.6 & ND & ND & ND & NR & ND & 1.5 & 1.6 & 2.6 & 1.7 & 3.0 & ND & ND \\
\hline $\mathrm{C}_{17: 1} \omega 7 c$ & ND & ND & ND & ND & ND & ND & ND & NR & ND & ND & ND & ND & ND & ND & ND & ND \\
\hline $\mathrm{C}_{17: 1} \omega 8 c$ & ND & ND & ND & ND & ND & ND & 9.3 & NR & ND & 0.4 & ND & ND & ND & 0.4 & 1.0 & 2.9 \\
\hline $\mathrm{C}_{18: 1} \omega 5 c$ & 0.5 & $\mathrm{ND}$ & 3.7 & 0.7 & ND & ND & ND & NR & 1.5 & 1.8 & $\mathrm{ND}$ & 1.0 & 0.8 & 1.0 & ND & 2.7 \\
\hline $\mathrm{C}_{18: 1} \omega 7 c$ & 33.9 & 45.7 & 37.8 & 51.3 & 67.3 & 73.1 & 70.5 & + & 59.9 & 67.0 & 64.8 & 41.4 & 46.0 & 40.5 & 56.0 & 73.1 \\
\hline $\mathrm{C}_{17: 0}$ cyclo & ND & ND & ND & ND & ND & ND & ND & NR & ND & ND & ND & ND & 0.4 & ND & ND & $\mathrm{ND}$ \\
\hline $\mathrm{C}_{19: 0} \omega 8 c$ cyclo & ND & ND & 1.0 & 0.8 & ND & ND & ND & NR & ND & ND & ND & ND & 0.7 & ND & ND & ND \\
\hline $\mathrm{C}_{16: 1} 2-\mathrm{OH}$ & ND & ND & ND & ND & ND & ND & ND & NR & ND & $\mathrm{ND}$ & ND & ND & 0.5 & ND & ND & ND \\
\hline $11-\mathrm{Me}-\mathrm{C}_{18: 1} \omega 7 c$ & ND & 6.3 & ND & ND & ND & ND & ND & NR & ND & ND & ND & ND & 3.5 & ND & ND & $\mathrm{ND}$ \\
\hline
\end{tabular}

${ }^{\star}$ Complete data for S. melonis is not available.

pyruvate, D-ribose, sucrose, sorbose, sucrose, D-xylose, L-alanine as sole carbon source but not adonitol, citrate, dulcitol, dextran, ethanolamine, fumaric acid, glucuronic acid, myo-inositol, lactose, lactic acid, D-levulose, D-maltose, D-mannitol, D-mannose, myristic acid, oxalic acid, raffinose, D-rhamnose, L-sorbose, sorbitol, succinate, trehalose, L-arginine, L-aspartic acid, L-aspargine, L-cysteine, L-glycine, L-glutamine, L-glutamic acid, L-histidine, L-leucine, L-lysine, L-methionine, L-phenylalanine and L-proline. Cells are sensitive to (per disc): azithromycin $(30 \mu \mathrm{g})$, bacitracin $(10 \mathrm{U})$, ceftriaxone $(30 \mu \mathrm{g})$, chloramphenicol $(30 \mu \mathrm{g})$, ciprofloxacin $(5 \mu \mathrm{g})$, doxycycline $(30 \mu \mathrm{g})$, gentamicin $(10 \mu \mathrm{g})$, novobiocin $(30 \mu \mathrm{g})$, rifampicin $(30 \mu \mathrm{g})$, tetracycline $(30 \mu \mathrm{g})$ and vancomycin $(30 \mu \mathrm{g})$, but resistant to aztreonam $(30 \mu \mathrm{g})$, carbenicillin $(100 \mu \mathrm{g})$, cephalothin $(30 \mu \mathrm{g})$, colistin $(10 \mu \mathrm{g})$, erythromycin $(2 \mu \mathrm{g})$, ethambutol $(50 \mu \mathrm{g})$, ethionamide $(30 \mu \mathrm{g})$, nitrofurantoin $(150 \mu \mathrm{g})$, penicillin $(10 \mu \mathrm{g})$, polymyxin B $(300 \mu \mathrm{g})$, streptomycin $(10 \mu \mathrm{g})$, sulfisoxazole $(300 \mu \mathrm{g})$, sulfathiazole $(300 \mu \mathrm{g})$ and trimethoprim $(5 \mu \mathrm{g})$. The fatty acids and their contribution to the total fatty acid content are listed in Table 2 . The major lipids present are phosphatidylglycerol (PG), cardiolipin
(DPG), phosphatidyldimethyl ethanolamine (PDE) and sphingoglycolipid (SGL).

The type strain, strain CP173- $2^{\mathrm{T}}\left(=\right.$ ATCC BAA- $1239^{\mathrm{T}}=$ DSM $17494^{\mathrm{T}}$ ), was isolated from a biological soil crust from the Colorado Plateau, USA.

\section{Description of Sphingomonas desiccabilis sp. nov.}

Sphingomonas desiccabilis (de.sic.ca'bi.lis. L. v. desiccare to dry up; L. suffix -abilis adjectival suffix expressing passive qualities; N.L. fem. adj. desiccabilis able to be dried, desiccable).

Colonies are yellow-pigmented, extremely mucoid, convex, round and smooth. Cells are Gram-negative, non-motile, small rods of $0.25 \mu \mathrm{m}$ in diameter and $0.5 \mu \mathrm{m}$ in length. Grows at $15-37^{\circ} \mathrm{C}$, with an optimum growth temperature of $25{ }^{\circ} \mathrm{C}$ and $\mathrm{pH}$ of 7 . Can tolerate up to $4 \% \mathrm{NaCl}$. The fatty acids and their contribution to the total fatty acid content are listed in Table 2. Major fatty acids and other metabolic characteristics are the same as those for Sphingomonas 
mucosissima $\mathrm{CP} 173-2^{\mathrm{T}}$, except the following: tests positive for lipase, gelatinase and $\beta$-galactosidase, negative in tests for urease and can reduce nitrate to nitrite. Can utilize glucuronic acid, D-maltose, D-mannose, raffinose, D-rhamnose and L-proline as sole carbon source, but not acetate, D-fructose, glycerol or L-alanine. Cells are resistant to gentamicin $(10 \mu \mathrm{g} /$ disc $)$.

The type strain, strain CP1D ${ }^{\mathrm{T}}\left(=\right.$ ATCC BAA $-1041^{\mathrm{T}}=\mathrm{DSM}$ $16792^{\mathrm{T}}$ ), was isolated from a biological soil crust from the Colorado Plateau, USA.

\section{Acknowledgements}

This research was funded by the National Science Foundation Biotic Surveys and Inventories grant 0206711, to F. G-P. We also thank Professor H. J. Busse and Professor J. H. Yoon for kindly providing the type strains of S. panni and S. dokdonensis and Marlene Garcia-Neuer and Kayla Urcheck for nomenclatural suggestions.

\section{References}

Belnap, J. \& Lange, O. L. (2001). Biological Soil Crusts: Structure, Function, and Management, Ecological Studies Series, vol. 150. Berlin, Germany: Springer.

Bowker, M. A., Reed, S. C., Belnap, J. \& Phillips, S. L. (2002) Temporal variation in community composition, pigmentation, and $\mathrm{F}(\mathrm{v}) / \mathrm{F}(\mathrm{m})$ of desert cyanobacterial soil crusts. Microb Ecol 43, 13-25.

Buonaurio, R., Stravato, V. M., Kosako, Y., Fujiwara, N., Naka, T., Kobayashi, K., Cappelli, C. \& Yabuuchi, E. (2002). Sphingomonas melonis sp. nov., a novel pathogen that causes brown spots on yellow Spanish melon fruits. Int J Syst Evol Microbiol 52, 2081-2087.

Busse, H. J., Denner, E. B. M., Buczolits, S., Salkinoja-Salonen, M., Bennasar, A. \& Kämpfer, P. (2003). Sphingomonas aurantiaca sp. nov., Sphingomonas aerolata sp. nov. and Sphingomonas faeni sp. nov., air- and dustborne and Antarctic, orange-pigmented, psychrotolerant bacteria, and emended description of the genus Sphingomonas. Int J Syst Evol Microbiol 53, 1253-1260.

Busse, H. J., Hauser, E. \& Kämpfer, P. (2005). Description of two novel species, Sphingomonas abaci sp. nov. and Sphingomonas panni sp. nov. Int J Syst Evol Microbiol 55, 2565-2569.

Collins, M. D., Pirouz, T., Goodfellow, M. \& Minnikin, D. E. (1977). Distribution of menaquinones in actinomycetes and corynebacteria. J Gen Microbiol 100, 221-230.

Denner, E. B. M., Kämpfer, P., Busse, H.-J. \& Moore, E. R. B. (1999). Reclassification of Pseudomonas echinoides Heumann 1962, 343 ${ }^{\mathrm{AL}}$, in the genus Sphingomonas as Sphingomonas echinoides comb. nov. Int J Syst Bacteriol 49, 1103-1109.

Denner, E. B. M., Paukner, S., Kämpfer, P., Moore, E. R. B., Abraham, W. R., Busse, H.-J., Wanner, G. \& Lubitz, W. (2001). Sphingomonas pituitosa sp. nov., an exopolysaccharide-producing bacterium that secretes an unusual type of sphingan. Int J Syst Evol Microbiol 51, 827-841.

Garcia-Pichel, F. (2002). Desert environments: biological soil crusts. In Encyclopedia of Environmental Microbiology, pp. 1019-1023. Edited by G. Bitton. New York: Wiley.

Gundlapally, S. R. \& Garcia-Pichel, F. (2006). The community and phylogenetic diversity of biological soil crusts in the Colorado Plateau studied by molecular fingerprinting and intensive cultivation. Microbial Ecol 52, 345-357.
Johnson, S. L., Budinoff, C. R., Belnap, J. \& Garcia-Pichel, F. (2005). Relevance of ammonium oxidation within biological soil crust communities. Environ Microbiol 7, 1-12.

Kimura, M. (1980). A simple method for estimating evolutionary rates of base substitutions through comparative studies of nucleotide sequences. J Mol Evol 16, 111-120.

Kumar, S., Tamura, K., Jakobsen, I. B. \& Nei, M. (2001). MEGA2: molecular evolutionary genetics analysis software. Bioinformatics 17, 1244-1245.

Lee, J. S., Shin, Y. K., Yoon, J. H., Takeuchi, M., Pyun, Y. R. \& Park, Y. H. (2001). Sphingomonas aquatilis sp. nov., Sphingomonas koreensis sp. nov. and Sphingomonas taejonensis sp. nov., yellow-pigmented bacteria isolated from natural mineral water. Int J Syst Evol Microbiol 51, 1491-1498.

Maruyama, T., Park, H. D., Ozawa, K., Tanaka, Y., Sumino, T., Hamana, K., Hiraishi, A. \& Kato, K. (2006). Sphingosinicella microcystinivorans gen. nov., sp. nov., a microcystin-degrading bacterium. Int J Syst Evol Microbiol 56, 85-89.

Nagy, M., Pérez, A. \& Garcia-Pichel, F. (2005). The prokaryotic diversity of biological soil crusts in the Sonoran Desert (Organ Pipe Cactus National Monument, AZ). FEMS Microbiol Ecol 54, 233-245.

Ohta, H., Hattori, R., Ushiba, Y., Mitsui, H., Ito, M., Watanabe, H., Tonosaki, A. \& Hattori, T. (2004). Sphingomonas oligophenolica sp. nov., a halo- and organo-sensitive oligotrophic bacterium from paddy soil that degrades phenolic acids at low concentrations. Int J Syst Evol Microbiol 54, 2185-2190.

Pal, R., Bala, S., Dadhwal, M., Kumar, M., Dhingra, G., Prakash, O., Prabagaran, S. R., Shivaji, S., Cullum, J. \& other authors (2005). Hexachlorocyclohexane-degrading bacterial strains Sphingomonas paucimobilis B90A, UT26 and Sp + having similar lin genes are three distinct species, Sphingobium indicum sp. nov.; Sphingobium japonicum sp. nov. and Sphingobium francense sp. nov. and reclassification of [Sphingomonas] chungbukensis as Sphingobium chungbukense comb. nov. Int J Syst Evol Microbiol 55, 1965-1972.

Pal, R., Bhasin, V. K. \& Lal, R. (2006). Proposal to reclassify [Sphingomonas] xenophaga Stolz et al. 2000 and [Sphingomonas] taejonensis Lee et al. 2001 as Sphingobium xenophagum comb. nov. and Sphingopyxis taejonensis comb. nov., respectively. Int J Syst Evol Microbiol 56, 667-670.

Pandey, K. K., Mayilraj, S. \& Chakrabarti, T. (2002). Pseudomonas indica sp. nov., a novel butane-utilizing species. Int $J$ Syst Evol Microbiol 52, 1559-1567.

Pollock, T. J. (1993). Gellan-related polysaccharides and the genus Sphingomonas. J Gen Microbiol 139, 1939-1945.

Reddy, G. S. N. \& Garcia-Pichel, F. (2005). Dyadobacter crusticola sp. nov., from biological soil crusts in the Colorado Plateau, USA, and emended description of the genus Dyadobacter Chelius and Tripplett 2000. Int J Syst Evol Microbiol 55, 1295-1299.

Reddy, G. S. N., Nagy, M. \& Garcia-Pichel, F. (2006). Belnapia moabensis gen. nov., sp. nov., an alphaproteobacterium from biological soil crusts in the Colorado Plateau, USA. Int J Syst Evol Microbiol 56, 51-58.

Stackebrandt, E. \& Goebel, B. M. (1994). Taxonomic note: a place for DNA-DNA reassociation and 16S rRNA sequence analysis in the present species definition in bacteriology. Int J Syst Bacteriol 44, 846-849.

Suresh, K., Reddy, G. S. N., Sengupta, S. \& Shivaji, S. (2004). Deinococcus indicus sp. nov., an arsenic-resistant bacterium from an aquifer in West Bengal, India. Int J Syst Evol Microbiol 54, 457-461. Takeuchi, M., Kawai, F., Shimada, Y. \& Yokota, A. (1993). Taxonomic study of polyethylene glycol-utilizing bacteria: emended description of the genus Sphingomonas and new descriptions of 
Sphingomonas macrogoltabidus sp. nov., Sphingomonas sanguis sp. nov. and Sphingomonas terrae sp. nov. Syst Appl Microbiol 16, 227-238.

Takeuchi, M., Sakane, T., Yanagi, M., Yamasato, K., Hamana, K. \& Yokota, A. (1995). Taxonomic study of bacteria isolated from plants: proposal of Sphingomonas rosa sp. nov., Sphingomonas pruni sp. nov., Sphingomonas asaccharolytica sp. nov., and Sphingomonas mali sp. nov. Int J Syst Bacteriol 45, 334-341.

Takeuchi, M., Hamana, K. \& Hiraishi, A. (2001). Proposal of the genus Sphingomonas sensu stricto and three new genera, Sphingobium, Novosphingobium and Sphingopyxis, on the basis of phylogenetic and chemotaxonomic analyses. Int J Syst Evol Microbiol 51, 1405-1417.

Tamaoka, J. (1986). Analysis of bacterial menaquinone mixtures by reverse-phase high-performance liquid chromatography. Methods Enzymol 123, 251-256.

Tamaoka, J., Katayama-Fujimura, Y. \& Kuraishi, H. (1983). Analysis of bacterial menaquinone mixture by high performance liquid chromatography. J Appl Bacteriol 54, 31-36.
Thompson, J. D., Higgins, D. G. \& Gibson, T. J. (1994). CLUSTAL W: improving the sensitivity of progressive multiple sequence alignment through sequence weighting, position-specific gap penalties and weight matrix choice. Nucleic Acids Res 22, 4673-4680.

Yabuuchi, E., Yano, I., Oyaizu, H., Hashimoto, Y., Ezaki, T. \& Yamamoto, H. (1990). Proposals of Sphingomonas paucimobilis gen. nov. and comb. nov., Sphingomonas parapaucimobilis sp. nov., Sphingomonas yanoikuyae sp. nov., Sphingomonas adhaesiva sp. nov., Sphingomonas capsulata comb. nov., and two genospecies of the genus Sphingomonas. Microbiol Immunol 34, 99-119.

Yeager, C. M., Kornosky, J. L., Housman, D. C., Grote, E. E., Belnap, J. \& Kuske, C. R. (2004). Diazotrophic community structure and function in two successional stages of biological soil crusts from the Colorado Plateau and Chihuahuan Desert. Appl Environ Microbiol 70, 973-983.

Yoon, H. J., Lee, M. H., Kang, S. J., Lee, S. Y. \& Oh, T. K. (2006). Sphingomonas dokdonensis sp. nov., isolated from soil. Int J Syst Evol Microbiol 56, 2165-2169. 